\title{
Metabolic responses of HeLa cells to silica nanoparticles by NMR- based metabolomic analyses
}

\author{
Jianghua Feng $\cdot$ Jinquan Li $\cdot$ Huifeng Wu $\cdot$ \\ Zhong Chen
}

Received: 20 November 2012/ Accepted: 16 January 2013/Published online: 26 January 2013

(C) Springer Science+Business Media New York 2013

\begin{abstract}
Silica nanoparticles are increasingly used in the biomedical fields due to their excellent solubility, high stability and favorable biocompatibility. However, despite being considered of low genotoxicity, their bio-related adverse effects have attracted particular concern from both the scientific field and the public. In this study, human cervical adenocarcinoma cells (HeLa line) were exposed to 0.01 or $1.0 \mathrm{mg} / \mathrm{mL}$ of hydrophilic silica nanoparticles. The ${ }^{1} \mathrm{H}$ NMR spectroscopy coupled with multivariate statistical analysis were used to characterize the metabolic variations of intracellular metabolites and the compositional changes of the corresponding culture media. At the early stage of silica nanoparticles-exposure, no obvious dose-effect of HeLa cell metabolome was observed, which implied that cellular stress-response regulated the metabolic variations of HeLa cell. Silica nanoparticles induced the increases of lipids including triglyceride, LDL, VLDL and lactate/alanine ratio and the decreases of alanine, ATP, choline, creatine, glycine, glycerol, isoleucine, leucine, phenylalanine, tyrosine, and valine, which involved in membrane
\end{abstract}

Electronic supplementary material The online version of this article (doi:10.1007/s11306-013-0499-8) contains supplementary material, which is available to authorized users.

J. Feng $(\bowtie) \cdot$ J. Li $\cdot$ Z. Chen

Fujian Provincial Key Laboratory of Plasma and Magnetic Resonance, Department of Electronic Science, Xiamen

University, Xiamen 361005, China

e-mail: jianghua.feng@xmu.edu.cn

$\mathrm{H} . \mathrm{Wu}$

Key Laboratory of Coastal Zone Environment Processes,

CAS, Shandong Provincial Key Laboratory of Coastal Zone

Environment Processes, Yantai Institute of Coastal Zone

Research, Chinese Academy of Sciences, Yantai 264003, China modification, catabolism of carbohydrate and protein, and stress-response. Subsequently, a complicated synergistic effect of stress-response and toxicological-effect dominated the biochemical process and metabolic response, which was demonstrated in the reverse changes of some metabolites including acetate, ADP, ATP, choline, creatine, glutamine, glycine, lysine, methionine, phenylalanine and valine between 6 and $48 \mathrm{~h}$ post-treatment of silica nanoparticles. The toxicological-effects induced by highdosage silica nanoparticles could be derived from the elevated levels of ATP and ADP, the utilization of glucose and amino acids and the production of metabolic endproducts such as glutamate, glycine, lysine, methionine, phenylalanine, and valine. The results indicated that it is important and necessary to pursue further the physiological responses of silica nanoparticles in animal models and human before their practical use. NMR-based metabolomic analysis helps to understand the biological mechanisms of silica nanoparticles and their metabolic fate, and further, it offers an ideal platform for establishing the bio-safety of existing and new nanomaterials.

Keywords Silica nanoparticles - Metabolomic analysis NMR spectroscopy $\cdot$ Nanomedicine $\cdot$ HeLa cell

\section{Introduction}

Because of their excellent solubility, high stability and favorable biocompatibility, silica nanoparticles $\left(\mathrm{SiO}_{2}-\mathrm{NPs}\right)$ are increasingly used in the biomedical research as delivery vehicles of therapeutic, diagnostic, biosensing and imaging agents (Roy et al. 2005; Tsai et al. 2009; $\mathrm{Gu}$ et al. 2010), the capsules of controlled release drug (Vivero-Escoto et al. 2010), or bone graft substitute 
(Ghanaati et al. 2010). However, based on their special size, composition, transportation and biodistribution in the body, the health-related risks of silica nanoparticles, such as silicosis, pulmonary fibrosis and lung cancer, have been found (Chen et al. 2008; Choi et al. 2008). Their biorelated adverse effects in living systems have attracted particular concern from both the scientific field and the public. Before promoting further applications of these nanomaterials in the biomedical field, systematic toxicological characterizations in living systems are critically needed. In the last decade, the cytotoxic effects induced by silica nanoparticles have been investigated in different cell models and animal models, and the original results indicated that the treatments with silica nanoparticles can alter the activities of the functional proteins and disrupt the structure of the cells (Gao et al. 2009; Lai et al. 2010; $\mathrm{Xu}$ et al. 2010). The potential risks of silica nanoparticles on human health have also been identified (Sharma et al. 2009; Han et al. 2011), but the molecular mechanisms to induce possible nanotoxicity and risks have not been fully understood, and the biological effects have not been described at the molecular level.

Because of the expense of in vivo animal models and public and governmental urging to develop alternative to animal testing, in vitro models may be more feasible and practical to assess the potential toxicological effects of nanomaterials and understand their basic mechanisms of physiological processes (Yang et al. 2005). Furthermore, understanding the cellular responses of silica nanoparticles is a good starting point and such investigation can provide holistic metabolic information on the responses of biological systems in the molecular level. In this regard, NMR-based metabolomic analysis on cell lines could elucidate the biological mechanism and interactions involved in cellular pathways, to screen promising drugs and to identify and validate new cellular targets. Such approach has been proven to be a valid and rapid in vitro screening method for detecting significant metabolite changes in response to a nanomaterial treatment according to our previous study (Feng et al. 2011).

In the present study, we applied an integrated metabolomic approach to systematically investigate the metabolic responses of HeLa cells to silica nanoparticles. The ${ }^{1} \mathrm{H}$ NMR spectroscopy coupled with pattern recognition analysis were used to characterize the metabolic profiles of both intracellular metabolism of HeLa cells and their culture media. The aims of the current investigation were to provide basic information on the underlying interaction of $\mathrm{SiO}_{2}$-NPs with biological systems and to provide an alternative methodology for the assessments of prior bioeffect study of the bio-nanomaterials.

\section{Materials and methods}

\subsection{Silica nanoparticles}

The silica nanoparticles $\left(\mathrm{SiO}_{2}\right.$-NPs) were synthesized by the sol-gel process as reported previously (Yu et al. 2005). The diameter of hydrophilic silica nanoparticles is $20 \pm 3 \mathrm{~nm}$. The as-synthesized samples were characterized by transmission electron microscopy (TEM) and fourier transform infrared spectroscopy (FTIR). The silica concentration in $\mathrm{SiO}_{2}$-NPs was determined by inductively coupled plasma-atomic emission spectrometry (ICP-AES). The obtained silica nanoparticles were resuspended in PBS solution before incubation with cells. Cells were then incubated with silica nanoparticles in the concentrations of 0.01 and $1.0 \mathrm{mg} \mathrm{SiO} \mathrm{O}_{2} / \mathrm{mL}\left(10^{6}\right.$ cells $)$.

\subsection{Incubation of HeLa cells with the silica nanoparticles}

Human cervical adenocarcinoma cells (HeLa line) were obtained from Yantai Institute of Coastal Zone Research, CAS (Yantai, China) and grown at $37{ }^{\circ} \mathrm{C}$ in a humidified atmosphere of $5 \% \mathrm{CO}_{2}$ in DMEM medium, supplemented with $10 \%(\mathrm{v} / \mathrm{v})$ fetal bovine serum, $1 \%$ sodium pyruvate, $2 \mathrm{mM}$ glutamine, $100 \mu \mathrm{g} / \mathrm{mL}$ streptomycin and $100 \mathrm{U} / \mathrm{mL}$ penicillin. Fresh stock of HeLa cells were seeded at a density of $3.0 \times 10^{5}$ cells $/ 75 \mathrm{~cm}^{2}$ flask in six identical sets (5 samples each). After 2 days, the growth medium was replaced with fresh growth medium and the cells were allowed to grow further for another 2 days during which the cells became confluent (approximately $10^{7}$ cells/flask). $100 \mu \mathrm{L}$ of silica nanoparticles in $\mathrm{pH}$ 7.0 PBS buffer was added to the cell culture flask for the incubation. The final concentrations of nanoparticles were $0,0.01$ or $1.0 \mathrm{mg} / \mathrm{mL}$. The cells were exposed continuously until harvest. After the addition of nanoparticles $6 \mathrm{~h}$ later, the cells in three sets of flasks (control, low- and high-dosage groups, five samples each group) were harvested by trypsinization with $1 \mathrm{~mL}$ trypsin solution. At $48 \mathrm{~h}$ after incubation with silica nanoparticles, the cells of all the other flasks (five samples each group) were harvested as above. All HeLa cells in trypsin solution were centrifuged at $1,000 \mathrm{~g}$ for $5 \mathrm{~min}$. The supernatants were discarded, and the cells were washed twice with ice-cold PBS $\left(4{ }^{\circ} \mathrm{C}, \mathrm{pH} 7.4\right) .1 .5 \mathrm{~mL}$ of cell culture medium for each sample was collected before harvesting cells and cleared by centrifugation (10 min, $12,000 \times g)$. During all of these treatments, the cells were incubated at $37{ }^{\circ} \mathrm{C}$ in a $5 \% \mathrm{CO}_{2}$ and humidified atmosphere. The cells and culture media collected were immediately snap frozen in liquid nitrogen and stored at $-80{ }^{\circ} \mathrm{C}$ until NMR analysis. 
The cytotoxicity of silica nanoparticles and cell conditions at the different time-points were monitored by Trypan blue staining and microscopic method.

\subsection{Assessment of cell viability using the MTT assay}

The in vitro cytotoxicity was measured by cell viability via MTT test which measured mitochondrial activity. The MTT assay is based on the reduction of MTT ((3-(4,5dimethylthiazol-2-yl)-2,5-diphenyltetrazolium bromide) by mitochondrial succinate dehydrogenase to form dark blue formazan crystals, in which only viable cells with active mitochondria can reduce significant amounts of MTT to formazan. For this assay, HeLa cells were seeded in a 96-well plate using $3 \times 10^{4}$ cells/well, and incubated for $24 \mathrm{~h}$, before adding various concentrations of silica nanoparticles to each well, and incubating the cells for a further 6 or $48 \mathrm{~h}$. After the incubation period, $20 \mu \mathrm{L}$ of MTT $(0.5 \mathrm{mg} / \mathrm{mL}$ in phosphate buffer, at $\mathrm{pH} 7.4)$ was added to each well and the cells were incubated for another $3 \mathrm{~h}$. After discarding the media, the formazan crystals were solubilized by $100 \mu \mathrm{L}$ of dimethyl sulfoxide (DMSO) per well and the absorbance was measured at $565 \mathrm{~nm}$ using a Bio-Rad microplate reader.

\subsection{Sample preparation and NMR experiments}

\subsection{1 ${ }^{1}$ H-high-resolution magic-angle-spinning (HRMAS) NMR spectroscopy}

For HRMAS ${ }^{1} \mathrm{H}$ NMR spectroscopic analysis, HeLa cell samples were removed from storage at $-80{ }^{\circ} \mathrm{C}$ to room temperature to defrost. The cell pellets (approximately $20 \mathrm{mg}$ ) were packed into separate $4 \mathrm{~mm}$ diameter zirconium oxide rotors with sufficient saline in $\mathrm{D}_{2} \mathrm{O}$ added to maintain a cellular osmolality and to provide a field-lock. All the HRMAS ${ }^{1} \mathrm{H}$ NMR spectra were recorded at $298 \mathrm{~K}$ on a Varian INOVA-600 NMR spectrometer equipped with a high-resolution MAS probe (Moka et al. 1997; Waters et al. 2000), operating at a ${ }^{1} \mathrm{H}$ frequency of $599.90 \mathrm{MHz}$. Cell samples were spun at $2.5 \mathrm{kHz}$ at the magic-angle $\left(54.7^{\circ}\right)$. A total of $15 \mathrm{~min}$ was allowed for the temperature equilibration before NMR acquisition. HRMAS ${ }^{1} \mathrm{H}$ NMR spectrum was acquired using a water-suppressed CarrPurcell-Meiboom-Gill (CPMG) spin-echo pulse sequence (recycle delay- $90^{\circ}-\left(\tau-180^{\circ}-\tau\right)_{\mathrm{n}}$-acquisition). The spectra were measured by using a spin-spin relaxation delay, $2 n \tau$, of $70 \mathrm{~ms}$ and water signal irradiation was applied during the recycle delay. Typically, 128 transients were acquired with $32 \mathrm{k}$ data points for each spectrum with a spectral width of $20 \mathrm{ppm}$, and the $90^{\circ}$ pulse length was adjusted to approximately $10 \mu$ s for $90^{\circ}$ pulse calibration individually.

\subsection{2 ${ }^{1}$ H NMR spectroscopy of cell culture medium}

NMR measurements of cell culture medium were carried out at $298 \mathrm{~K}$ in random order on a Varian INOVA-600 NMR spectrometer. For each sample, the standard NOESYPR1D pulse sequence (recycle delay- $90-\mathrm{t}_{1}-90-\mathrm{t}_{\mathrm{m}}{ }^{-}$ 90 -acquisition) was employed with the irradiations at the water frequency during the recycle delay of $2 \mathrm{~s}$ and the mixing time of $100 \mathrm{~ms}$ to suppress the water signal. Typically, sixty-four transients were acquired with $32 \mathrm{k}$ data points with a spectral width of $20 \mathrm{ppm}$. And the $90^{\circ}$ pulse length which was adjusted to about $10 \mu$ s for each sample and $\mathrm{t}_{1}$ was set to $3 \mu \mathrm{s}$.

\subsection{Spectral processing and multivariate data analysis}

The NMR data for both cells and culture media were converted into Bruker format from Varian format before data processing. For all 1D ${ }^{1} \mathrm{H}$ NMR spectra, free induction decays (FIDs) were multiplied by an exponential function with a $0.3 \mathrm{~Hz}$ (for the cells) or $1.0 \mathrm{~Hz}$ (for the media) linebroadening factor prior to Fourier transformation. All the ${ }^{1} \mathrm{H}$ NMR spectra were manually phased and baseline-corrected using Topspin 3.0 software (Bruker Biospin, Germany). The spectra of cells and media were referenced to the internal lactate methyl resonance at $\delta 1.33$. The spectra regions $\delta 8.50-0.50$ (for the cells) or $\delta 9.00-0.50$ (for the media) were automatically divided into integral segments of equal width (0.005 ppm) using AMIX (V3.9, Bruker Biospin). In the case of HRMAS NMR spectra, the regions of $\delta 5.27-4.20$ were removed to eliminate the effects of imperfect water suppression. For the NMR spectra of the media, the regions of $\delta 6.89-4.29$ and $\delta 3.89-3.83$, $\delta 3.20-2.70$ and $\delta 3.70-3.64, \delta 1.22-1.17$ were excluded to eliminate the residual water, HEPES and ethanol signals, respectively. The integrated data were normalized to the total sum of the spectrum before multivariate statistical analysis to give the same total integration value for each spectrum before data analysis.

Multivariate statistical analysis was carried out with the software package SIMCA-P + (V11.0, Umetrics, Sweden). Principal component analysis (PCA) was performed by using a mean-centered scaling approach, and the data were visualized in the form of the principal component (PC) scores plots to identify general metabolic trends and possible outliers. To improve the separation due to silica nanoparticles treatment and to uncover the statistically significant metabolite variations related to silica nanoparticles-exposure, sample classes were modeled using orthogonal projection to latent structure with discriminant analysis (OPLS-DA) algorithm at a unit variance (UV) scaling approach. The results were visualized in the forms of scores plots to show the group clusters and loadings 
plots to show the NMR variables associated to clustering. The loadings in the coefficient plots were calculated back from the coefficients incorporating the weight of the variables in order to enhance interpretability of the OPLS-DA model. In the coefficient-coded loadings plots, biochemical components responsible for the differences between samples can be extracted from the corresponding loadings with the weight of the variable contributing to the discrimination. The coefficient plots were generated with MATLAB (V7.1, the Mathworks Inc., Natwick, USA) scripts with some in-house modifications and were color-coded with absolute value of coefficients (r). In our case, a hot color (i. e. red) corresponds to the metabolites being highly related to the discrimination between pair-wise classes, while a cool color (i. e. blue) corresponds to no significance. In our study, a correlation coefficient of $|r|>0.811$ was used as the cutoff value for the statistical significance based on the discrimination significance at the level of $p=0.05$ and $d f$ $($ degree of freedom $)=4$, which was determined according to the test for the significance of the Pearson's productmoment correlation coefficient. The validation of the model was conducted using five fold cross validation and the cross-validation parameter $\mathrm{R}^{2}$ and $\mathrm{Q}^{2}$ values were used to assess the amount of variation represented by the PCs and robustness of the model. Additional, all models were further validated by permutation tests (permutation numbers $=100$ ) for the corresponding projection to latent structure with discriminant analysis (PLS-DA) models (Trygg et al. 2007). The $\mathrm{R}^{2}$ in the permutated plot described how well the data fit the derived model, whereas $\mathrm{Q}^{2}$ describes the predictive ability of the derived model and provides a measure of the model quality. If the maximum value of $Q_{\max }^{2}$ from the permutation test was smaller than or equal to the $\mathrm{Q}^{2}$ of the real model, the model was regarded as a predictable model. Similarly, the $\mathrm{R}^{2}$ value and difference between the $R^{2}$ and $Q^{2}$ were used to evaluate the possibility of over-fitted models.

\section{Results and discussion}

3.1 Viability of HeLa cells in response to the exposure of silica nanoparticles

The cytotoxicity of the nanoparticles was detected by measuring the cell proliferation rate, cell death, and death patterns (necrosis and apoptosis) after the nanoparticles treatments. No obvious cell damage and death was found in both low- and high-dosage groups at $6 \mathrm{~h}$ post-treatment and low-dosage $(0.01 \mathrm{mg} / \mathrm{mL})$ group at $48 \mathrm{~h}$ post-treatment, while HeLa cell viabilities were significantly decreased to $76.9 \%(p<0.05)$ in high-dosage $(1.0 \mathrm{mg} / \mathrm{mL})$ group compared with the corresponding controls at $48 \mathrm{~h}$ post-treatment with silica nanoparticles (data not shown). Previous results show no significantly toxic effects due to the silica nanoparticles at the molecular and cellular levels below a concentration of $0.1 \mathrm{mg} / \mathrm{mL}$ (Jin et al. 2007), which was confirmed by our present observation. However, even when no cell damage or death may be apparent following the nanoparticles-exposure, metabolic changes may result from stress-response and cellular function (Mbeh et al. 2012). Beyond basic silica nanoparticles dose-effect behavior, the compositions of culture media also contribute to the ambiguity of cell interactions (Maiorano et al. 2010). Therefore, it is important to monitor the changes of cellular metabolome and composition of culture media in order to verify the cytotoxicity of HeLa cell in response to the exposure of silica nanoparticles.

\subsection{NMR profile of HeLa cells and corresponding} culture media

The analysis of cellular metabolome could directly provide the information of cellular metabolic response on the environmental stimuli, and the metabolites compositional changes in culture media not only reflect the substrate utilization and production but also indirectly provide the information of the physiological status and phenotype of the corresponding cells.

${ }^{1} \mathrm{H}$ NMR spectra of HeLa cells and corresponding culture medium were acquired in order to observe the metabolic effects of silica nanoparticles on metabolomic variations of HeLa cells and compositional changes of the culture media. Primary resonances in the ${ }^{1} \mathrm{H}$ NMR spectra of cell and culture media samples were assigned to individual metabolites (Table S1) according to the literature data (Bailey et al. 2003; Bundy et al. 2006; Micceli et al. 2006; Akhtar et al. 2007; Feng et al. 2011) and confirmed by public NMR database (Human Metabolome Database V3.0, see www.hmdb.ca) and in-house developed NMR database. Predominated by various lipids and choline-like metabolites, the NMR spectra of HeLa cells also contained numerous resonances from other metabolites including essential amino acids (isoleucine, leucine, valine, lysine, tyrosine, and phenylalanine), non-essential amino acids (alanine, methionine, glycine, and glutamate), TCA intermediates (lactate, citrate, and succinate) and ADP and ATP. The NMR spectra of culture media were characterized by a source of nutrient substrates, such as various amino acids, glucose, and choline, to provide the entire necessary elements for the cellular growth, and various metabolic end-products and intermediates, such as the intermediates of glycolysis and TCA (pyruvate, acetate, lactate and succinate), the waste metabolites (TMAO and formate) and several carboxylic acids (isobutyrate and $\alpha$-hydroxyisobutyrate). Following the treatment of $\mathrm{SiO}_{2}-\mathrm{NPs}$, 
NMR profile of HeLa cells demonstrated an obvious metabolic differences between control and treated cells (Fig. 1d, e), such as the higher level of phenylalanine, tyrosine and choline and the lower level of lactate, acetate and phosphocholine (PC) in the treated group than the controls, indicating that silica nanoparticles induced alterations in the metabolism of HeLa cells. The consuming of the nutrients such as glucose and several kinds of amino acids and the producing of metabolic end-products such as pyruvate, lactate, formate and acetate were also observed in the NMR spectra of culture media following the treatment of $\mathrm{SiO}_{2}$-NPs (Fig. 1a-c).

\subsection{Silica nanoparticles induced metabolomic variations of HeLa cells}

To get the detailed metabolic information of HeLa cells following the treatment of silica nanoparticles, multivariate data analysis was employed to analyze the metabolic variations induced by silica nanoparticles and identify the possible metabolic pathways involved.

Principal component analysis (PCA) from NMR data of HeLa cells revealed the obvious time- and dose-responses (Fig. 2a) of $\mathrm{SiO}_{2}$-NPs. We noticed the metabolic differences between control (C6) and treated cells (L6 and H6)

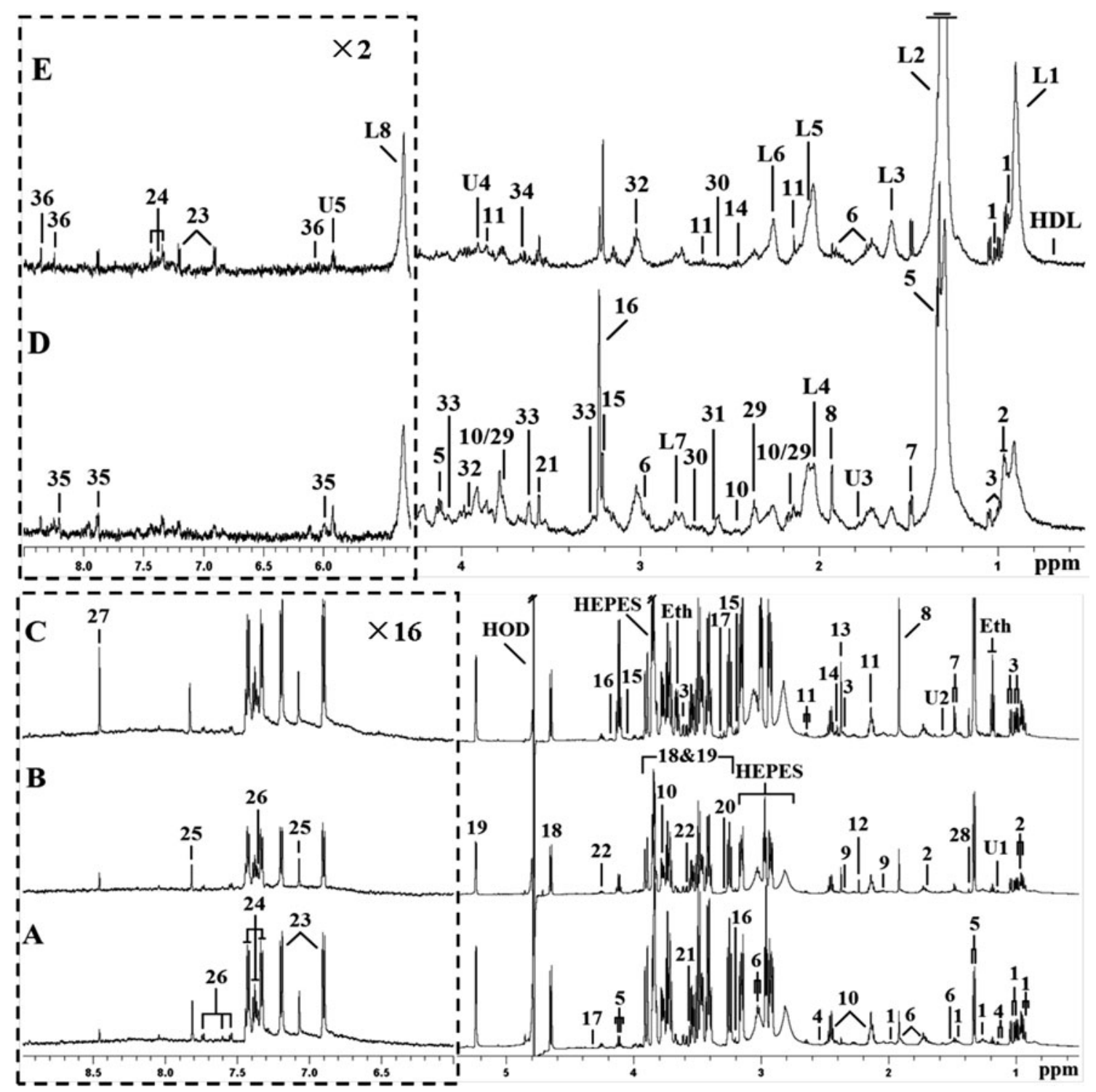

Fig. 1 Representative $600 \mathrm{MHz}{ }^{1} \mathrm{H}-\mathrm{NOESY}$ NMR spectra $(\delta 0.5-5.4$, 86.0-9.0) of cell growth medium from a control b $6 \mathrm{~h}$ and $\mathbf{c} 48 \mathrm{~h}$ post-treatment with low-dosage silica nanoparticles and ${ }^{1} \mathrm{H}$-MAS CPMG NMR spectra $(\delta 0.5-4.3, \delta 5.2-8.5)$ of HeLa cells d pre- and e $48 \mathrm{~h}$ post-treatment with high-dosage silica nanoparticles. The aromatic regions (in the dashed boxes) were magnified two time (cells) or sixteen times (medium) and scaled in a different chemical shift expansion compared with corresponding aliphatic regions for the purpose of clarity. The metabolites numbered were assigned in Table $\mathrm{S} 1$ of supplementary materials 
and the metabolic similarity between low- (L6) and highdosage (H6) groups at $6 \mathrm{~h}$ post-treatment. A similar metabolic trajectory was displayed between low- and highdosage groups with the incubation time, however, an obvious dose-related metabolic profile was observed at $48 \mathrm{~h}$ post-treatment (Fig. $2 \mathrm{a}$ ).

To get an insight into the types of metabolites responsible for the class separation, the orthogonal projection to latent structure with discriminant analysis (OPLS-DA) was conducted with the corresponding NMR data from the pairwise cell groups. The OPLS-DA scores and corresponding loadings plots derived from the NMR data of pair-wise groups were shown in Fig. 3 and Fig. S1. The relative changes in metabolites with significant correlation coefficients between groups (Table 1) providing the biochemical alterations in HeLa cells following the treatment of silica nanoparticles. In order to evaluate the validity of these models, permutation test (permutation number $=100$ ) was conducted and five fold cross-validation parameters, $\mathrm{Q}^{2}$ and $\mathrm{R}^{2}$, were calculated from corresponding PLS-DA models of the pair-wise groups (Fig. S2).

No obvious dose-effect of HeLa cell metabolome was observed at $6 \mathrm{~h}$ post-treatment though some subtle differences existed between low- and high-dosage groups (Fig. 2a), implying that metabolic variations came mainly from cellular stress-response at the early stage of $\mathrm{SiO}_{2}$ NPs-exposure. Such information could be confirmed from the metabolomic trends (Fig. S1; Table 1), where only the metabolic difference from few metabolites was observed between low- and high-dosage groups. A previous study also showed stress-related cellular response induced by $\mathrm{SiO}_{2}$-NPs at an early stage (Mohamed et al. 2011). In our study, the stress-related metabolic variations included the increases of lipids including triglyceride, LDL and VLDL and the decreases of alanine, ATP, choline, creatine, glycine, glycerol, isoleucine, leucine, phenylalanine, tyrosine, and valine. The lipid components of the cell membrane
Fig. 3 OPLS-DA scores plots (left panel) and corresponding coefficient plots (middle and right panels) derived from the ${ }^{1} \mathrm{H}$ HRMAS CPMG NMR spectra of HeLa cells obtained from different pair-wise groups. The color maps show the significance of metabolite variations between the two classes. Peaks in the positive direction indicate metabolites that are more abundant in the dosed groups than control group. Consequently, metabolites that are more abundant in the control group are presented as peaks in the negative direction. $C$ control groups, $L$ low-dosage groups, $H$ high-dosage groups, $66 \mathrm{~h}$ post-treatment, $4848 \mathrm{~h}$ post-treatment with $\mathrm{SiO}_{2}$ nano-particles. Keys: Ace acetate, Ala alanine, Cit citrate, $\mathrm{Cr}$ Creatine, Gln glutamine, Glu glutamate, GL Glycerol, Gly glycine, Ileu isoleucine, Lac lactate, Leu leucine, Lys lysine, m-I myo-inositol, Met methonine, $P C$ phosphocholine, Phe phenylalanine, Tyr tyrosine, Val valine (Color figure online)

form the permeability barrier (Berg et al. 2002), and the increased level of lipids implied that the cellular membrane permeability was probably increased over $6 \mathrm{~h}$ exposures to silica nanoparticles, which was confirmed by cellular membrane permeability test in the similar study (Mohamed et al. 2011) and the decreased level of choline and glycine (Griffin et al. 2001; Feng et al. 2011). It is known that the alterations of the cellular membrane permeability take effects on the physiological condition of the cells (Mingeot-Leclercq et al. 1995), consequently, the decreased levels of amino acid such as alanine, glycine, isoleucine, leucine, phenylalanine, tyrosine, and valine indicated an inhibitor of protein catabolism in response to $\mathrm{SiO}_{2}-\mathrm{NPs}$ exposure. The previous studies (Oliveira et al. 2011; Rato et al. 2012) have proven that the ratio of lactate/alanine can be served to be an indicative of cellular redox state. A lower lactate/alanine ratio implies the lower reductive/ higher oxidative state for the cells. In our study, a higher lactate/alanine ratio was observed in the high-dosage group $(30.3 \pm 5.8$ vs $13.4 \pm 8.8$ of control, $p<0.01)$ at $6 \mathrm{~h}$ post-treatment of silica nanoparticles, which indicated a higher reductive/lower oxidative state of the HeLa cells. The reduced ATP in our study may be related to oxidative phosphorylation in mitochondria (Robinson et al. 2012),
Fig. 2 PCA scores plots based on the ${ }^{1} \mathrm{H}$ NMR spectra of HeLa cells (a) and cell growth media (b) following the time- and dose-responses of silica nanoparticles. $C$ control groups, $L$ low-dosage groups, $H$ high-dosage groups, $66 \mathrm{~h}$ post-treatment, $4848 \mathrm{~h}$ posttreatment with silica nanoparticles (Color figure online)

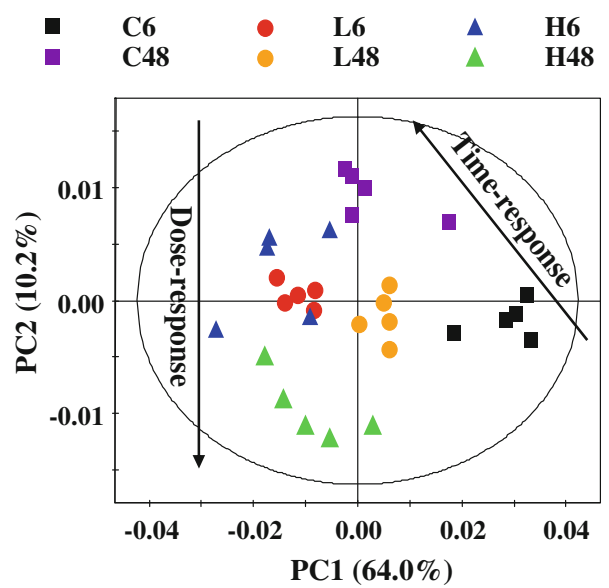

(A)

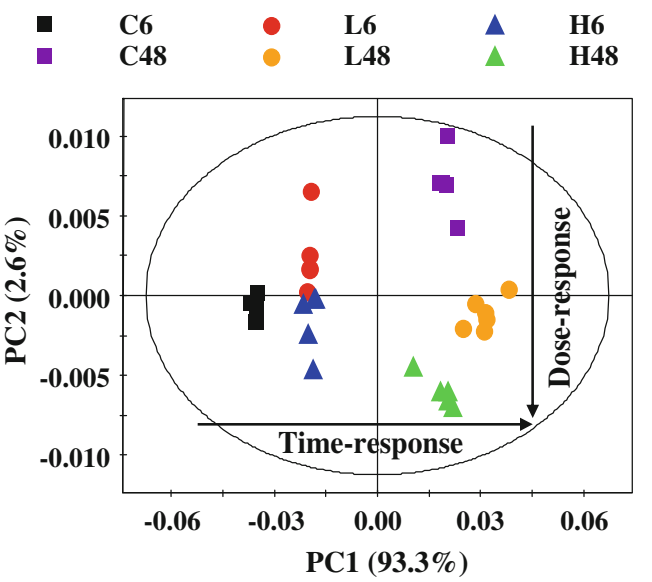

(B) 


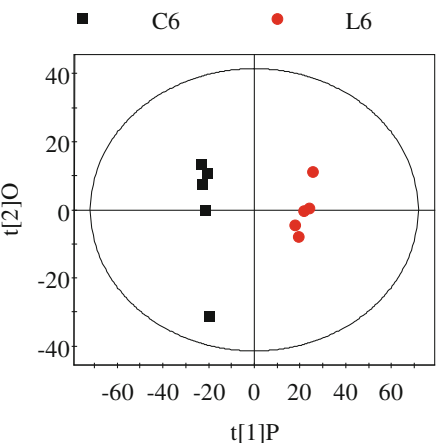

$\mathrm{R}^{2} \mathrm{X}=51.8 \%, \mathrm{Q}^{2}=0.923$

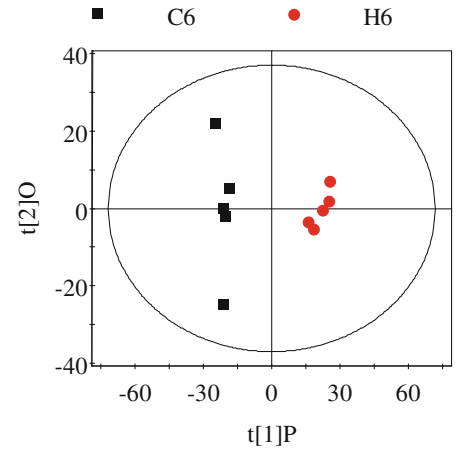

$\mathrm{R}^{2} \mathrm{X}=54.7 \%, \mathrm{Q}^{2}=0.924$

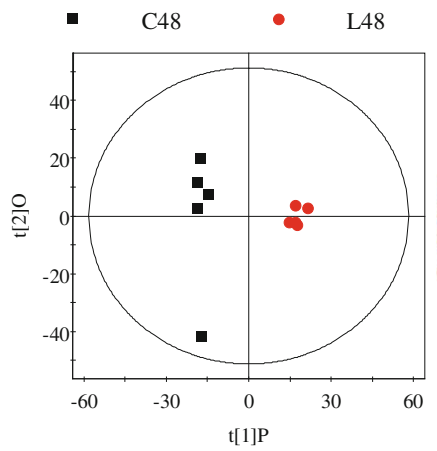

$\mathrm{R}^{2} \mathrm{X}=46.2 \%, \mathrm{Q}^{2}=0.762$

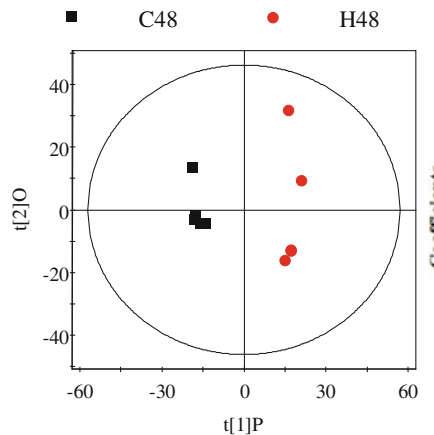

$\mathrm{R}^{2} \mathrm{X}=42.7 \%, \mathrm{Q}^{2}=0.757$
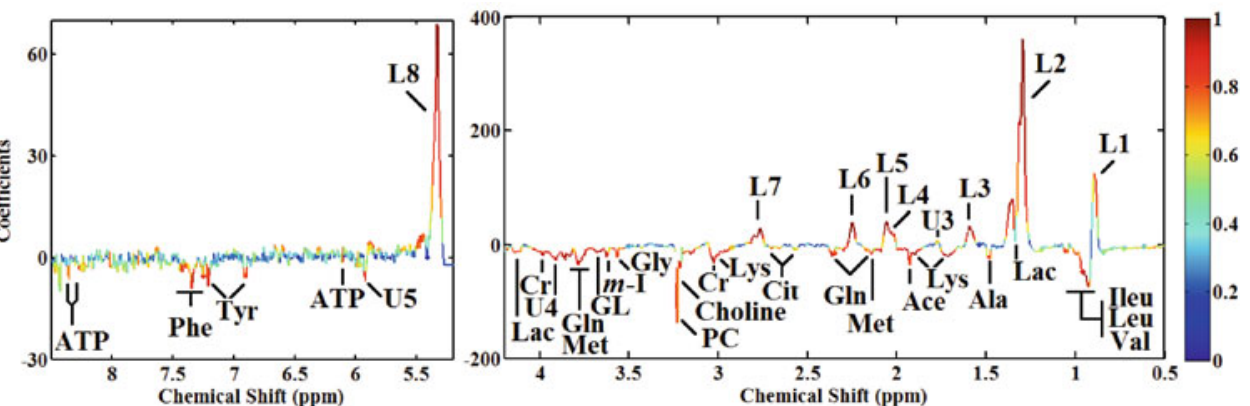

(A)
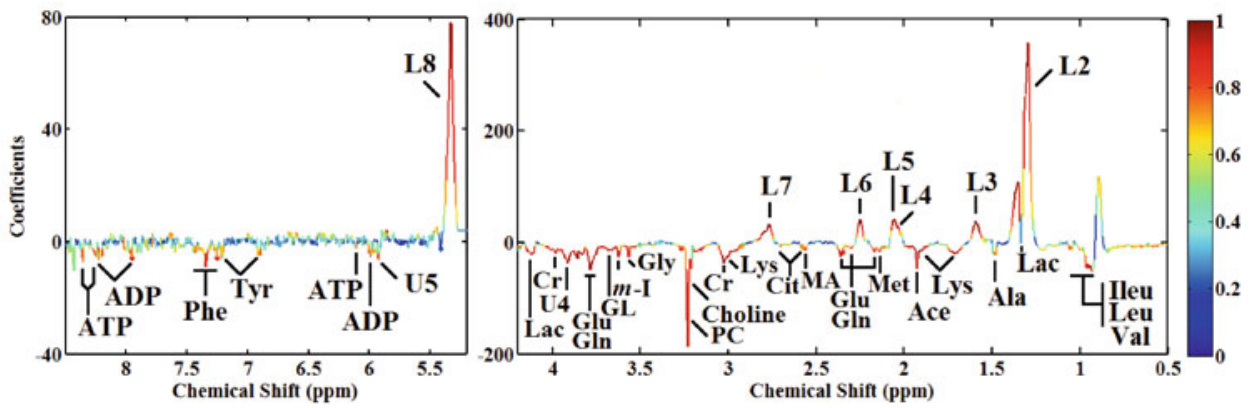

(B)
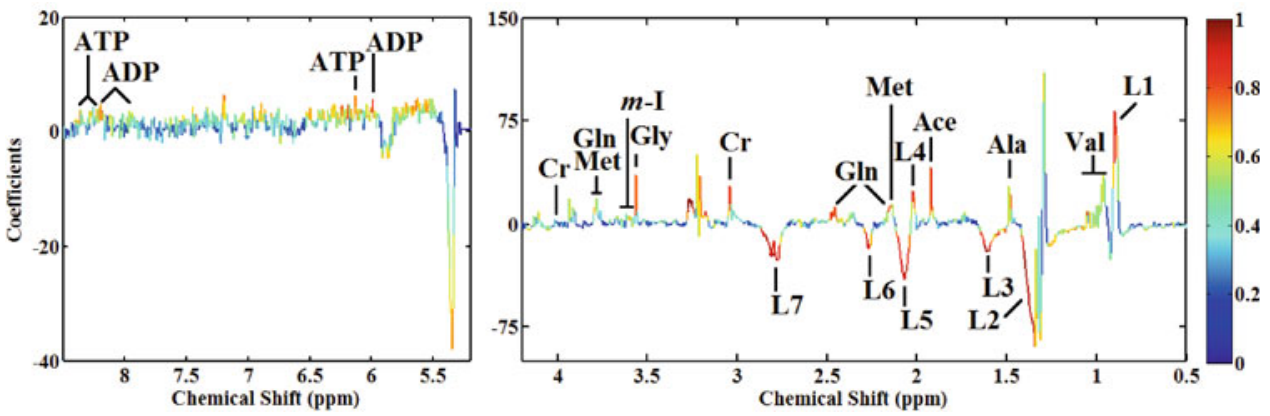

(C)
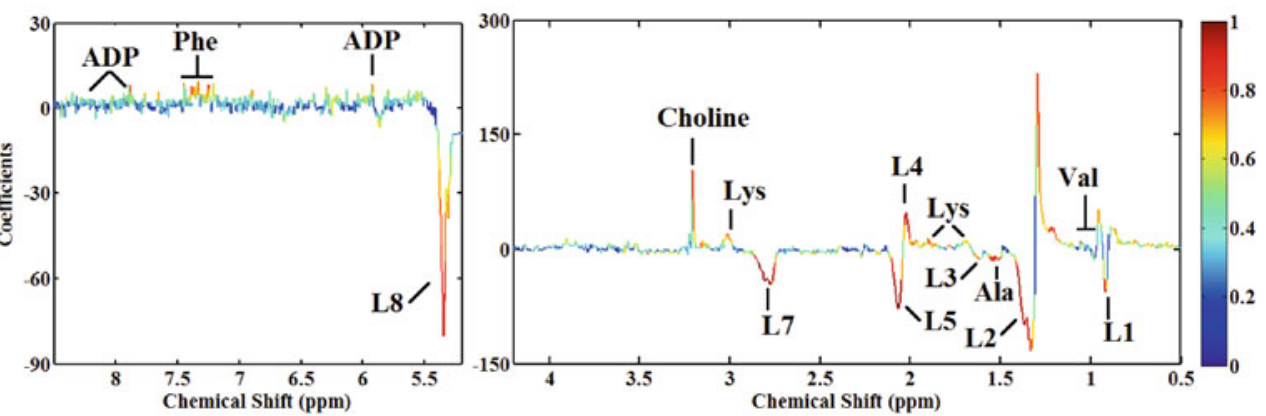

(D) 
which was promoted by the activation of transcription factor-2 (ATF-2) (Mohamed et al. 2011), a member of the basic transcription factor family that regulates the expression of genes in response to various stress signals (Gupta et al. 1995). However, such conclusion needs to be further tested in the possible animal experiment.

A dose-dependent spectral change, characterized by decreased resonance intensities from acetate, ADP, citrate, glutamate, glutamine, lactate, lysine, methylamine, methionine, myo-inositol and phosphocholine (PC), was also observed during the exposure of silica nanoparticles especially at the early stage (Table 1). It means that stimulation of a higher dose of silica nanoparticles led to enhanced
Fig. 4 OPLS-DA scores plots (left panel) and corresponding coefficient plots (middle and right panels) derived from the ${ }^{1} \mathrm{H}$ NOESY NMR spectra of cell growth media obtained from different pair-wise groups. The color maps show the significance of metabolite variations between the two classes. Peaks in the positive direction indicate metabolites that are more abundant in the dosed groups than control group. Consequently, metabolites that are more abundant in the control group are presented as peaks in the negative direction. $C$ control groups, $L$ low- $\&$ $\mathrm{H}$ high-dosage groups, $66 \mathrm{~h}$ post-treatment, $4848 \mathrm{~h}$ post-treatment with $\mathrm{SiO}_{2}$ nano-particles. Keys: Ace acetate, Act actone, Ala alanine, For formate, Glc Glucose, Gln glutamine, GPC glycerophosphocholine, $H I B$ a-hydrxoyisobutyrate, His histidine, $I B$ isobutyrate, Ileu isoleucine, Lac lactate, Leu leucine, Lys lysine, Met methonine, PC phosphocholine, Phe phenylalanine, Pro proline, Py Pyruvate, Suc succinate, TMAO trimethylamine N-oxide, Try tryptophan, Tyr tyrosine, $\mathrm{Val}$ valine (Color figure online)

Table 1 Overview of metabolite variations in the HeLa cells observed between different $\mathrm{SiO}_{2}-\mathrm{NPs}_{\text {administrations }}$

\begin{tabular}{|c|c|c|c|c|c|c|}
\hline \multirow[t]{2}{*}{ Metabolites } & \multicolumn{6}{|l|}{$\mathrm{r}^{\mathrm{a}}$} \\
\hline & C6-L6 & C6-H6 & L6-H6 & C48-L48 & C48-H48 & L48-H48 \\
\hline Acetate & -0.972 & -0.975 & -0.844 & 0.857 & - & - \\
\hline ADP & - & -0.947 & -0.935 & 0.932 & 0.858 & -0.925 \\
\hline Alanine & -0.893 & -0.863 & - & 0.841 & -0.849 & - \\
\hline ATP & -0.984 & -0.981 & - & 0.842 & - & 0.838 \\
\hline Choline & -0.901 & -0.941 & - & - & 0.815 & - \\
\hline Citrate & -0.884 & -0.880 & -0.932 & - & - & 0.835 \\
\hline Creatine & -0.943 & -0.938 & - & 0.916 & - & 0.879 \\
\hline Glutamine & -0.951 & -0.968 & -0.874 & 0.915 & - & 0.934 \\
\hline Glutamate & -0.951 & -0.968 & -0.874 & - & - & 0.918 \\
\hline Glycine & -0.867 & -0.894 & - & 0.874 & - & - \\
\hline Glycerol & -0.988 & -0.979 & - & - & - & - \\
\hline Isoleucine & -0.977 & -0.964 & - & - & - & -0.942 \\
\hline L1: VLDL\&LDL $\mathrm{CH}_{3}-\left(\mathrm{CH}_{2}\right)_{\mathrm{n}}-$ & 0.842 & - & - & 0.886 & -0.909 & -0.889 \\
\hline L2: VLDL\&LDL $\overline{C H}_{3}-\left(\mathrm{CH}_{2}\right)_{\mathrm{n}}-$ & 0.991 & 0.975 & - & -0.955 & -0.975 & -0.815 \\
\hline L3: Triglyceride, $-\mathrm{CH}_{2}-\mathrm{CH}_{2}-\mathrm{C}=\mathrm{O}$ & 0.984 & 0.995 & 0.835 & -0.973 & -0.933 & - \\
\hline L4:Triglyceride, $-\mathrm{CH}_{2}-\mathrm{CH}=\mathrm{CH}-$ & 0.974 & 0.937 & - & 0.885 & 0.936 & -0.916 \\
\hline L5: Triglyceride, $-\mathrm{CH}_{2}-\mathrm{CH}=\mathrm{CH}-$ & 0.989 & 0.977 & - & -0.929 & -0.965 & 0.931 \\
\hline L6: Triglyceride, $-\mathrm{CH}_{2}-\mathrm{CH}_{2}-\mathrm{C}=\mathrm{O}$ & 0.996 & 0.994 & - & -0.908 & - & - \\
\hline L7: Triglyceride, $=\mathrm{CH}-\mathrm{CH}_{2}-\mathrm{CH}=$ & 0.987 & 0.992 & 0.834 & -0.969 & -0.985 & 0.988 \\
\hline L8: Triglyceride, $-\mathrm{CH}=\mathrm{CH}-$ & 0.987 & 0.982 & - & - & -0.889 & 0.905 \\
\hline Lactate & -0.926 & -0.955 & -0.898 & - & - & - \\
\hline Leucine & -0.944 & -0.892 & - & - & - & 0.819 \\
\hline Lysine & -0.990 & -0.992 & -0.894 & - & 0.867 & -0.947 \\
\hline Methylamine & - & -0.852 & -0.944 & - & - & 0.907 \\
\hline Methionine & -0.886 & -0.912 & -0.856 & 0.867 & - & 0.899 \\
\hline myo-Inositol & -0.940 & -0.957 & -0.951 & 0.976 & - & 0.977 \\
\hline $\mathrm{PC}$ & -0.930 & -0.902 & -0.906 & - & - & - \\
\hline Phenylalanine & -0.983 & -0.980 & - & - & 0.868 & -0.831 \\
\hline Tyrosine & -0.992 & -0.968 & - & - & - & -0.930 \\
\hline Valine & -0.936 & -0.886 & - & 0.822 & 0.882 & 0.923 \\
\hline
\end{tabular}

$C$ control groups, $L$ low-dosage groups, $H$ high-dosage groups, 66 h post-treatment, $4848 \mathrm{~h}$ post-treatment with $\mathrm{SiO}_{2}-\mathrm{NPs}$

${ }^{a}$ Correlation coefficients, positive and negative signs indicate positive and negative correlation in the concentrations, respectively. The correlation coefficient of $|\mathrm{r}|>0.811$ was used as the cutoff value for the statistical significance based on the discrimination significance at the level of $p=0.05$ and $d f$ (degree of freedom) $=4$. " "-" means the correlation coefficient $|r|$ is less than the cutoff value 


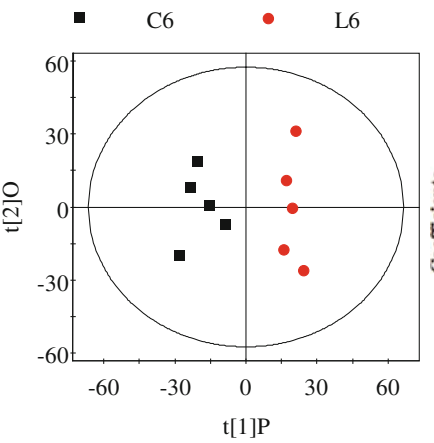

$\mathrm{R}^{2} \mathrm{X}=84.6 \%, \mathrm{Q}^{2}=0.873$

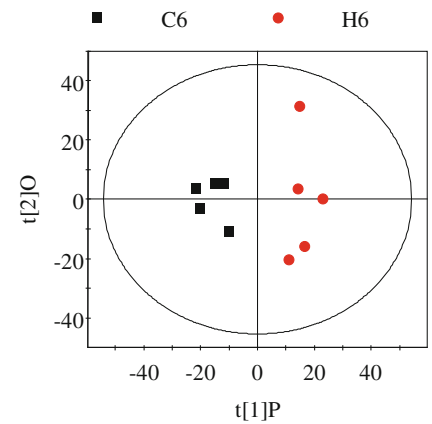

$\mathrm{R}^{2} \mathrm{X}=63.7 \%, \mathrm{Q}^{2}=0.803$

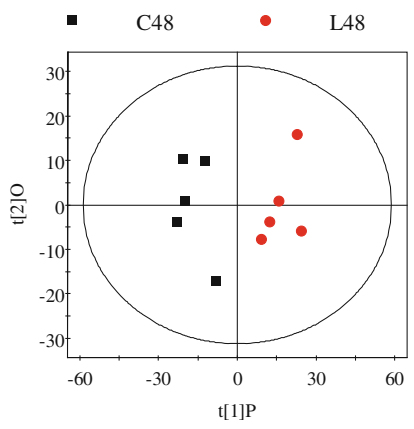

$\mathrm{R}^{2} \mathrm{X}=58.0 \%, \mathrm{Q}^{2}=0.664$

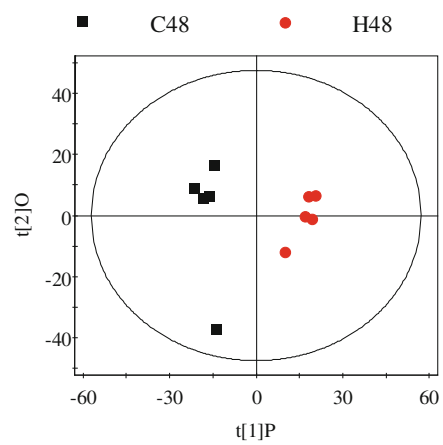

$\mathrm{R}^{2} \mathrm{X}=63.7 \%, \mathrm{Q}^{2}=0.833$
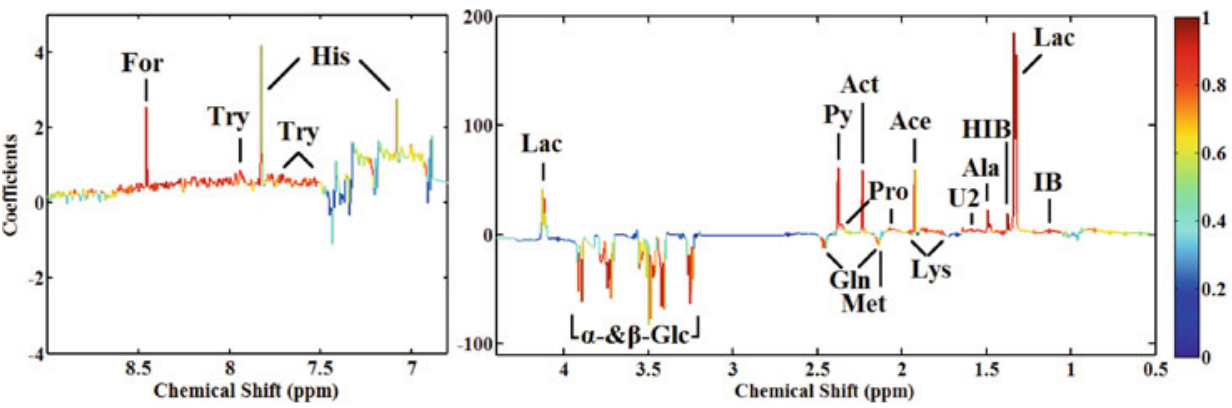

(A)
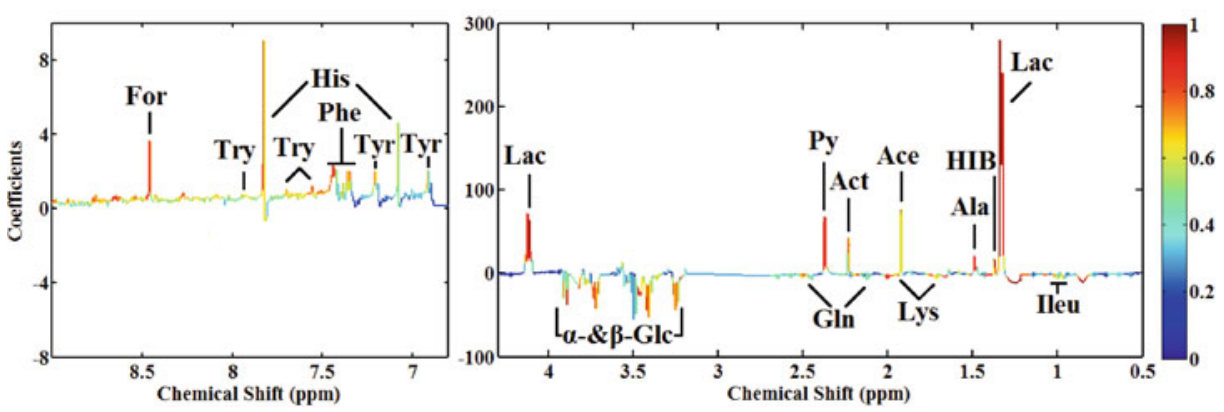

(B)
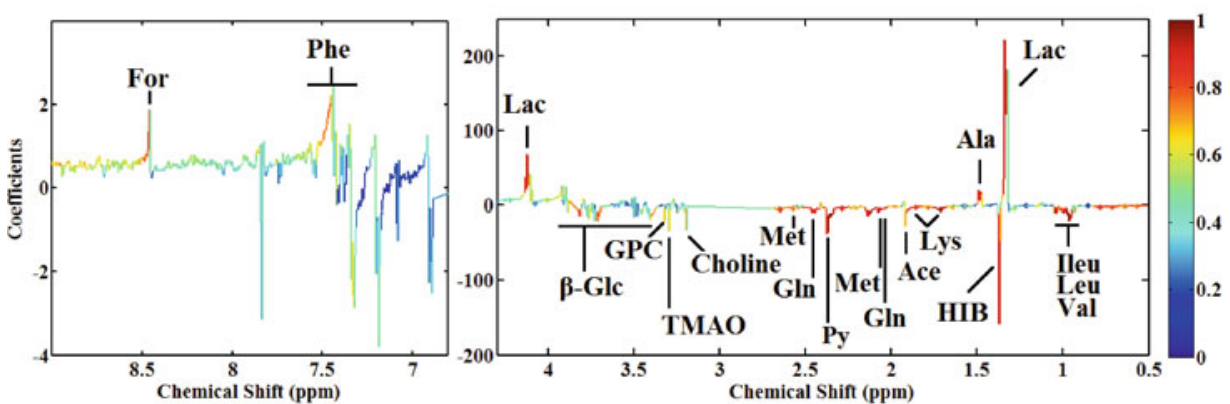

(C)
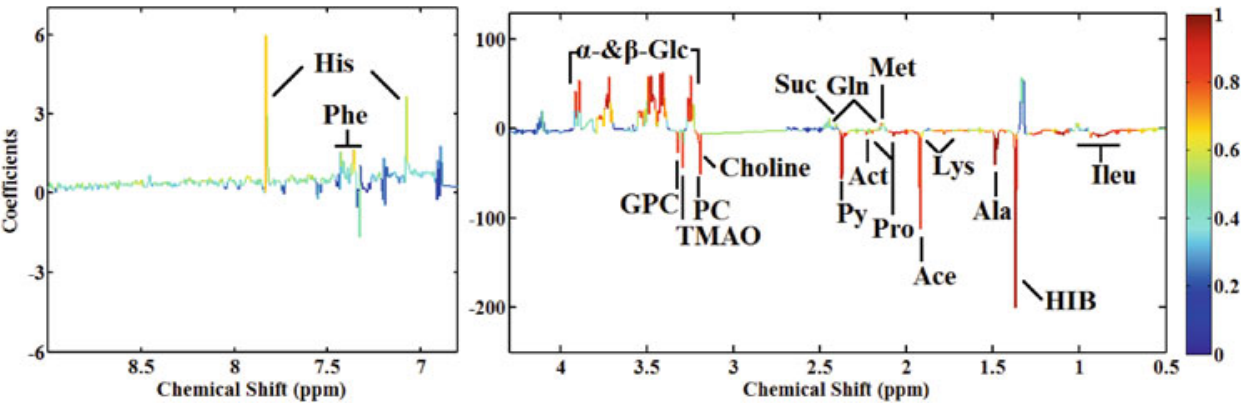

(D) 
Table 2 Overview of metabolite variations in the cell medium observed between different $\mathrm{SiO}_{2}-\mathrm{NPs}_{\text {administrations }}$

\begin{tabular}{|c|c|c|c|c|c|c|}
\hline \multirow[t]{2}{*}{ Metabolites } & \multicolumn{6}{|l|}{$\mathrm{r}^{\mathrm{a}}$} \\
\hline & C6-L6 & C6-H6 & L6-H6 & C48-L48 & C48-H48 & L48-H48 \\
\hline Acetate & 0.974 & 0.960 & - & -0.820 & -0.939 & -0.943 \\
\hline Acetone & 0.948 & 0.886 & -0.902 & - & -0.916 & - \\
\hline Alanine & 0.963 & 0.936 & -0.817 & 0.921 & -0.983 & -0.996 \\
\hline Choline & - & - & - & -0.875 & -0.950 & -0.908 \\
\hline Formate & 0.970 & 0.906 & - & 0.858 & - & -0.814 \\
\hline Glutamine & - & -0.884 & 0.955 & -0.984 & 0.812 & 0.975 \\
\hline Glycine & - & - & - & - & - & - \\
\hline GPC & - & - & - & -0.946 & -0.974 & - \\
\hline Histidine & 0.946 & 0.976 & - & - & 0.823 & - \\
\hline Isoleucine & - & -0.983 & -0.944 & -0.963 & -0.962 & 0.989 \\
\hline Isobutyrate & 0.853 & - & -0.919 & - & -0.967 & -0.966 \\
\hline Lactate & 0.972 & 0.988 & 0.842 & 0.935 & - & -0.905 \\
\hline Leucine & - & - & - & -0.977 & - & 0.988 \\
\hline Lysine & -0.949 & -0.938 & -0.953 & -0.966 & -0.924 & 0.986 \\
\hline Methoinine & -0.806 & - & - & -0.921 & 0.925 & 0.976 \\
\hline $\mathrm{PC}$ & - & - & - & - & -0.898 & -0.837 \\
\hline Phenylalanine & - & 0.866 & 0.933 & 0.812 & 0.812 & - \\
\hline Proline & 0.884 & - & -0.901 & -0.973 & -0.941 & 0.879 \\
\hline Pyruvate & 0.970 & 0.969 & - & -0.954 & -0.988 & -0.824 \\
\hline TMAO & - & - & - & -0.945 & -0.965 & -0.854 \\
\hline Tryptophan & 0.936 & 0.849 & - & - & - & - \\
\hline Valine & - & - & -0.851 & -0.963 & - & 0.955 \\
\hline$\alpha$-Glucose & -0.953 & -0.906 & 0.951 & - & 0.986 & 0.982 \\
\hline$\alpha$-Hydroxyisobutyrate & 0.981 & 0.967 & -0.948 & -0.953 & -0.979 & -0.927 \\
\hline$\beta$-Glucose & -0.968 & -0.919 & 0.951 & -0.841 & 0.973 & 0.983 \\
\hline
\end{tabular}

$C$ control groups, $L$ low-dosage groups, $H$ high-dosage groups, $66 \mathrm{~h}$ post-treatment, $4848 \mathrm{~h}$ post-treatment with $\mathrm{SiO}_{2}$-NPs

${ }^{a}$ Correlation coefficients, positive and negative signs indicate positive and negative correlation in the concentrations, respectively. The correlation coefficient of $|\mathrm{r}|>0.811$ was used as the cutoff value for the statistical significance based on the discrimination significance at the level of $p=0.05$ and $d f$ (degree of freedom) $=4$. " $-"$ means the correlation coefficient Irl is less than the cutoff value

stress-response including cellular membrane modification (derived from myo-inositol and PC) (Griffin et al. 2003), catabolism of carbohydrate and protein (derived from intermediates of TCA cycle and amino acids), and oxidative stress-response (derived from $\mathrm{ADP}$ and the lactate/ alanine ratio), consequently, a dose-response involved.

With the exposure of silica nanoparticles, a complicated synergistic effect was produced from the stress- and doseresponses at $48 \mathrm{~h}$ post-treatment. Silica nanoparticles induced the reverse changes of some metabolites including acetate, ADP, ATP, choline, creatine, glutamine, glycine, lysine, methionine, phenylalanine and valine between $6 \mathrm{~h}$ and $48 \mathrm{~h}$ post-treatment of silica nanoparticles (Table 1). Because the significantly toxicological effect was observed in the high-dosage group at $48 \mathrm{~h}$ post-treatment of silica nanoparticles, it will consequentially take effect on the cellular metabolisms over the exposure time. We noticed the elevated levels of ATP and ADP in the higher-dosage groups, which were associated with the initiation of apoptosis and cell death (Nishida et al. 2012) as visualized by viability of HeLa cells. As a response, the increased levels of some amino acids including glutamate, glycine, lysine, methionine, phenylalanine, and valine were observed, indicating high-dosage silica nanoparticles promoted the catabolism of protein and reduced protein synthesis.

\subsection{Compositional changes of culture media due to the treatment of silica nanoparticles}

PCA was also performed on NMR data of culture media of HeLa cells treated with silica nanoparticles (Fig. 2b). The scores plot showed marked difference in the composition of culture media between $6 \mathrm{~h}$ and $48 \mathrm{~h}$ post-treatment with silica nanoparticles in each pair-wise groups (Fig. 2b) with a similar metabolic trend. Following the silica nanoparticlesexposure, a clear dose-dependent response was observed in 
the composition of culture media at $48 \mathrm{~h}$ post-treatment. A clear difference can be observed in the media composition between the control and dosed groups at $6 \mathrm{~h}$ post-treatment, however, a close metabolic profile was displayed between high- and low-dosage groups, which was reasonably in response to the metabolic changes of HeLa induced by silica nanoparticles.

The same strategy was utilized to analyze NMR data from culture media in the pair-wise groups to obtain the detailed information of consumption of nutrients and production of metabolic end-products. These OPLS-DA models were validated using five fold cross validation strategy and rigorous permutation tests of the corresponding PLS-DA models (Fig. S4). The values of $R^{2} Y$ and $Q^{2}$ and the results of permutation tests indicated the validity of the models. OPLS-DA was performed with the NMR data of the pair-wise groups to identify the metabolites associated with such separations in the culture media following the exposure of silica nanoparticles (Figs. 4 and S3). And the metabolites with a significant contribution to the doseor time-classifications were listed in Table 2.

At the early stage of silica nanoparticles-exposure, glucose and amino acids utilization, with the production of metabolic end-products and intermediates, were characterized by high glycolytic activities under variations in energy and glucose supply. Lactate and acetate release is closely correlated with the variance of the glucose utilization due to the mainly cytosolic pyruvate production from glycolysis. Furthermore, alanine is released as an energy source from the alanine-glucose cycle after the formation of pyruvate from glycolysis (Mazurek and Eigenbroadt 2003). The obvious stress-response, i. e. the elevation of acetate, formate, histidine, pyruvate and tryptophan accompanied with the level decrease of methionine, and a dose-dependent effect, i. e. the elevation of acetone, alanine, isobutyrate (IB), lactate, phenylalanine, proline, and $\alpha$-hydroxyisobutyrate (HIB) and the level decrease of glutamine, isoleucine, lysine and glucose, could be derived from the analyses of compositional changes of culture media. Glucose and some amino acids including lysine, methionine, and isoleucine were more obviously consumed than others (Table 2), which may be due to the selective consumption of the nutritional materials by HeLa cells in response to growth needs and silica nanoparticles-exposure.

Similarly, the synergistic effect of stress-response and dose-effect was also observed due to the different metabolic profile of some metabolites, such as acetate, lactate, lysine, proline, valine, HIB, isoleucine and glucose in different dosage groups and at different time-points. However, it is obvious that the dose-effect dominated the compositional changes of culture media due to the treatment of silica nanoparticles at $48 \mathrm{~h}$ post-exposure, which was displayed by the dose-dependent metabolic changes of acetate, alanine, choline, IB, PC, pyruvate, TMAO and HIB. A reduced consumption of primary nutrients including glucose, methionine, glutamine, and alanine was observed in the higher-dosage groups compared with the controls, which was closely related to the cellular apoptosis and death induced by high-dosage silica nanoparticles, indicating an adaptive response to silica nanoparticles induced decrease in the energy demand of the HeLa cells.

\section{Conclusions}

Based on our results, we can conclude that silica nanoparticles induced the cellular metabolic responses involved in a complicated synergistic effect of stress-response and toxicological-effect. At the early stage of silica nanoparticles-exposure, cellular stress-response regulated the metabolic variations of HeLa cell, which involved in membrane modification, catabolism of carbohydrate and protein, and oxidative stress-response. Subsequently, the synergistic effect of stress-response and dose-effect induced by silica nanoparticles dominated the variations of cell metabolome and compositional changes of culture media. The toxicological effects induced by high-dosage silica nanoparticles and the information of glucose and amino acids utilization and the production of metabolic end-products could be derived from the metabolomic analysis of cell metabolomic variations and compositional changes. The preliminary results suggested that it is urgently needed to systematically pursue their potential toxicological effects in animal models and human before promoting further application of these nanomaterials in the biomedical fields. NMR-based metabolomic analysis provides a much better mechanistic understanding of silica nanoparticles and their metabolic fate, and further, it offers an ideal platform for establishing the bio-safety of existing and new nanomaterials.

Acknowledgments We acknowledge the financial supports from the National Natural Science Foundation of China (20605025, 21175099, 81272581) and the Fundamental Research Funds for the Central Universities (2011121046). This work was carried out on the Xiamen Research Platform on Systems Biology of Metabolic Disease.

\section{References}

Akhtar, S. V., Singh, R. K., Jadegoud, Y., Dhole, T. N., Ayyagari, A., \& Gowda, G. A. N. (2007). In vitro $1 \mathrm{H}$ NMR studies of RD human cell infection with Echovirus 11. NMR in Biomedicine, 20, 422-428.

Bailey, N. J. C., Oven, M., Holmes, E., Nicholson, J. K., \& Zenk, M. H. (2003). Metabolomic analysis of the consequences of cadimium exposure in silence cucubalus cell cultures via $1 \mathrm{H}$ NMR spectroscopy and chemometrics. Phytochemistry, 62, 851-858. 
Berg, J. M., Tymochzko, J. L., \& Stryer, L. (2002). Biochemistry (5th ed.). New York: Freeman.

Bundy, J. G., Iyer, N. G., Gentile, M. S., Hu, D. E., Kettunen, M., Maia, A. T., et al. (2006). Metabolic consequences of p300 gene deletion in human colon cancer cells. Cancer Research, 66, 7606-7614.

Chen, Z., Meng, H., Xing, G., Yuan, H., Zhao, F., Gao, X., et al. (2008). Age-related differences in pulmonary and cardiovascular responses to $\mathrm{SiO}_{2}$ nanoparticles inhalation: Nanotoxicity has susceptible population. Environmental Science and Technology, 42, 8985-8992.

Choi, M., Cho, W. S., Han, B. S., Cho, M., Kim, S. Y., Yi, J. Y., et al. (2008). Transient pulmonary fibrogenic effect induced by intratracheal instillation of ultrafine amorphous silica in $\mathrm{A} / \mathrm{J}$ mice. Toxicology Letters, 182, 97-101.

Feng, J., Zhao, J., Hao, F., Chen, C., Bhakoo, K., \& Tang, H. (2011). NMR-based metabonomics analyses of the effects of ultrasmall superparamagnetic particles of iron oxide (USPIO) on macrophage metabolism. Journal of Nanoparticle Research, 13, 2049-2062.

Gao, A., Song, S., Wang, D., Peng, W., \& Tian, L. (2009). Effect of silicon dioxide on expression of poly (ADP-ribose) polymerase mRNA and protein. Cell Biology International, 33, 749-754.

Ghanaati, S. M., Thimm, B. W., Unger, R. E., Orth, C., Kohler, T., Barbeck, M., et al. (2010). Collagen-embedded hydroxylapatitebeta-tricalcium phosphate-silicon dioxide bone substitute granules assist rapid vascularization and promote cell growth. Biomedical Materials, 5, 25004.

Griffin, J. L., Mann, C. J., Scott, J., Shoulders, C. C., \& Nicholson, J. K. (2001). Choline containing metabolites during cell transfection: An insight into magnetic resonance spectroscopy detectable changes. FEBS Letters, 509, 263-266.

Griffin, J. L., Pole, J. C., Nicholson, J. K., \& Carmichael, P. L. (2003). Cellular environment of metabolites and a metabonomics study of tamoxifen in endometrial cells using gradient high resolution magic angle spinning 1H NMR spectroscopy. Biochimica et Biophysica Acta, 1619, 151-158.

Gu, L., Park, J. H., Duong, K. H., Ruoslahti, E., \& Sailor, M. J. (2010). Magnetic luminescent porous silicon microparticles for localized delivery of molecular drug payloads. Small (Weinheim an der Bergstrasse, Germany), 6, 2546-5252.

Gupta, S., Campbell, D., Derijard, B., \& Davis, R. J. (1995). Transcription factor AFT2 regulation by the JNK signal transduction pathway. Science, 267, 389-393.

Han, B., Guo, J., Abrahaley, T., Qin, L., Wang, L., Zheng, Y., et al. (2011). Adverse effect of nano-silicon dioxide on lung function of rats with or without ovalbumin immunization. PLOS ONE, 6, e17236.

Jin, Y., Kannan, S., Wu, M., \& Zhao, J. X. (2007). Toxicity of luminescent silica nanoparticles to living cells. Chemical Research in Toxicology, 20, 1126-1133.

Lai, J. C., Ananthakrishnan, G., Jandhyam, S., Dukhande, V. V., Bhushan, A., Gokhale, M., et al. (2010). Treatment of human astrocytoma U87 cells with silicon dioxide nanoparticles lowers their survival and alter their expression of mitochondrial and cell signaling proteins. International Journal of Nanomedicine, 5, $715-723$.

Maiorano, G., Sabella, S., Sorce, B., Brunetti, V., Malvindi, M. A., Cingolani, R., et al. (2010). Effects of cell culture media on the dynamic formation of protein-nanoparticle complexes and influence on the cellular response. ACS Nano, 4, 7481-7491.

Mazurek, S., \& Eigenbroadt, E. (2003). The tumor metabolome. Anticancer Research, 23, 1149-1154.

Mbeh, D. A., França, R., Merhi, Y., Zhang, X. F., Veres, T., Sacher, E., et al. (2012). In vitro biocompatibility assessment of functionalized magnetite nanoparticles: Biological and cytotoxicological effects. Journal of Biomedical Materials Research, Part A, 100A, 1637-1646.

Micceli, A. T., Miccheli, A., Di Clemente, R., Valerio, M., Coluccia, P., Bizzari, M., et al. (2006). NMR-based metabolic profiling of human hepatoma cells in relation to cell growth by culture media analysis. Biochimica et Biophysica Acta, 1760, 1723-1731.

Mingeot-Leclercq, M. P., Brasseur, R., \& Schanck, A. (1995). Molecular-parameters involved in aminoglycoside nephrotoxicity. Journal of Toxicology and Environment Health, 44, 263-300.

Mohamed, B. M., Verma, N. K., Prina-Mello, A., Williams, Y., Davies, A. M., Bakos, G., et al. (2011). Activation of stressrelated signaling pathway in human cells upon $\mathrm{SiO}_{2}$ nanoparticles exposure as an early indicator of cytotoxicity. Journal of Nanobiotechnology, 9, 29.

Moka, D., Vorreuther, R., Schicha, H., Spraul, M., Humpfer, E., Lipinski, M., et al. (1997). Magic angle spinning proton nuclear magnetic resonance spectroscopic analysis of intact kidney tissue samples. Analytical Communications, 34, 107-109.

Nishida, K., Nakatani, T., Ohishi, A., Okuda, H., Higashi, Y., Matsuo, T., et al. (2012). Mitochondrial dysfunction is involved in P2X7 receptor-mediated neuronal cell death. Journal of Neurochemistry, 122, 1118-1128.

Oliveira, P. F., Alves, M. G., Rato, L., Silva, J., Sá, R., Barros, A., et al. (2011). Influence of 5 $\alpha$-dihydroxtestosterone and $17 \beta$-estradiol on human Sertoli cells metabolism. International Journal of Andrology, 34, e612-e620.

Rato, L., Alves, M. G., Socorro, S., Carvalho, R. A., Cavaco, J. E., \& Oliveira, P. F. (2012). Metaoblic modulation induced by oestradiol and DHT in immature rat Sertoli cells cultured in vitro. Bioscience Reports, 32, 61-69.

Robinson, G. L., Dinsdale, D., MacFarlane, M., \& Cain, K. (2012). Switching from aerobic glycolysis to oxidative phosphorylation modulates the sensitivity of mantle cell lymphoma cells to TRAIL. Oncogene, 31, 4996-5006.

Roy, I., Ohulchanskyy, T. Y., Bharali, D. J., Pudavar, H. E., Mistretta, R. A., Kaur, N., et al. (2005). Optical tracking of organically modified silica nanoparticles as DNA carriers: a nonviral, nanomedicine approach for gene delivery. Proceedings of the National Academy of Sciences of the United States of America, 102, 279-284.

Sharma, H. S., Patnaik, R., Sharma, A., Sjöquist, P. O., \& Lafuente, J. V. (2009). Silicon dioxide nanoparticles $\left(\mathrm{SiO}_{2}, 40-50 \mathrm{~nm}\right)$ exacerbate pathophysiology of traumatic spinal cord injury and deteriorate functional outcome in the rat. An experimental study using pharmacological and morphological approaches. Journal of Nanoscience and Nanotechnology, 9, 4970-4980.

Trygg, J., Holmes, E., \& Lundstedt, T. (2007). Chemometrics in metabonomics. Journal of Proteome Research, 6, 469-479.

Tsai, C. P., Chen, C. Y., Huang, Y., Chang, F. H., \& Mou, C. Y. (2009). Monoclonal antibody-functionalized mesoporous silica nanoparticles (MSN) for selective targeting breast cancer cells. Journal of Materials Chemistry, 19, 5737-5743.

Vivero-Escoto, J. L., Slowing, I. I., Trewyn, B. G., \& Lin, V. S. Y. (2010). Mesoporous silica nanoparticles for intracellular controlled drug delivery. Small (Weinheim an der Bergstrasse, Germany), 6, 1952-1967.

Waters, N. J., Garrod, S., Farrant, R. D., Haselden, J. N., Connor, S. C., \& Connelly, J. (2000). High-resolution magic angle spinning ${ }^{1} \mathrm{H}$ NMR spectroscopy of intact liver and kidney: Optimization of sample preparation procedures and biochemical stability of tissue during spectral acquisition. Analytical Biochemistry, 282, 16-23.

Xu, Z., Wang, S. L., \& Gao, H. W. (2010). Effects of nano-sized silicon dioxide on the structures and activities of three functional proteins. Journal of Hazardous Materials, 180, 375-383. 
Yang, M. S., Yu, L. C., \& Gupta, R. C. (2005). Analysis of multiple metabolomic subset in vitro: Methodological considerations. Toxicology Mechanisms and Methods, 15, 29-32.
Yu, K., Guo, Y., Ding, X., Zhao, J., \& Wang, Z. (2005). Synthesis of silica nanocubes by sol-gel method. Materials Letters, 59, 4013-4015. 